\title{
Ontology-Based Process Modeling Using eTOM and ITIL
}

\author{
Zhenning Shangguan, Zhipeng Gao and Kai Zhu \\ State Key Laboratory of Networking and Switching Technology, Beijing University of Posts \\ and Telecommunications, Beijing 100876, P.R.China \\ Shangguan.zhenning@gmail.com gaozhipeng@bupt.edu.cn zhukai@live.com
}

\begin{abstract}
With recently accelerated shifting trends from device-oriented to process-oriented service management, large portions of business organizations are now on their way of adopting standards and best-practices such as the eTOM and the ITIL to model and manage their diverse business and operational processes. Although there are various process/workflow definition formalisms to orchestrate these processes mentioned above, the rapid development and emerging demands for process automation and interoperability requires systematic modeling methodology and increased semantic information. To achieve this goal, a comprehensive analysis about two commonly accepted process frameworks - the eTOM and the ITIL - is presented in this paper first. Then, further studies concerning their mapping relationship and integrated application of these two process frameworks are carried out, which forms the basis of our process modeling methodology. We also propose an ontologybased process information model based on the preceding methodology, for the purpose of semantic richness. Finally, a novel and ontology-based business process management architecture is given to provide an application scenario and thus, to demonstrate the correctness and feasibility of the proposal in this paper.
\end{abstract}

Keywords: Business process modeling, eTOM, ITL, Ontology, OWL-S

\section{INTRODUCTION}

Over the recent years, the IT/ICT (Information, Communication and Technology) industry has been tremendously facilitated by the rapid evolution and expansion of the converged data and network services provided by the global telecommunication infrastructure. At the same time, these IT/ICT service providers have also been confronted with the formidable challenges of how to configure, provide and manage their business processes in an efficient and customer-oriented fashion. Although the overall productivity and quality of the IT/ICT enterprises have generally increased over the past years, huge waves of complaints from the customer side seem never to diminish. It is still time-consuming to implement and put into practice new business processes or change existing business processes according to customer needs. It seems that these companies always fail to keep pace with the rapid speed of emerging business changes. 
Clearly, there is a critical need for improvement. In fact, serious efforts concerning BPM (Business Process Management) from various parties, with the participation of international standardization organizations, academic institutions and consulting agents, etc., are now being carried out. Among all the contributions these parties have made, eTOM [1] and ITIL are two prevailing process management frameworks which are widely accepted guidelines in their own industry.

eTOM (Enhanced Telecom Operations Map) is part of the NGOSS (Next Generation Operational Support Systems) initiative proposed by the TMF (TeleManagement Forum). eTOM is a comprehensive business process framework to guide the development and management of key processes within any telecommunication service providers. It provides such guidance by offering a catalogue of standard terms and descriptions, with its scope spreads among multiple hierarchical levels. Since starting in 1999, eTOM has gradually added strategic, marketing, and product lifecycle planning and Enterprise process elements. One of eTOM's objectives is to aid the end-to-end automation of business and operations processes by using the eTOM process framework for its entire value chain, including, for example, the service providers, customers, the software/hardware vendors, and system integrators. eTOM has been adopted as ITU-T International Recommendation - known, in 2004, as M.3050.

ITIL (It Infrastructure Library) is the most well-known best practice and the defacto standard of IT Service Management (ITSM) developed by the Central Computer and Telecommunication Agency (CCTA) of the Britain Government and then adopted and customized by many companies in the IT industry as their internal IT practice. Since its first publication in 1980's, ITIL has gone through several minor restructuring and renaming which lead to the current ITIL Version 2. Among all of the topics covered in this new ITIL release, the most important content is the two "core titles", namely "Service Delivery" and "Service Support". The set of best practice guidelines and frameworks contained in ITIL will ensure quick delivery of reliable, consistent and of the highest quality.

In spite of all the endeavors mentioned above, service providers are now still facing with one critical dilemma: how to facilitate the integration and interoperability between heterogeneous industrial information environments [2]. Lessons from experience seem to indicate that the only way to overcome this difficulty is to express business process knowledge in a "global language" that can be spoken and understood by all participants. Such kind of "global language" needs syntax, and semantics of concepts, relationships, constraints and support for reasoning based on existent models. This is exactly what an ontology provides.

This paper presents a modeling methodology which is essentially two-fold: combining eTOM and ITIL to analyze composite business processes, and then define these processes based on semantic web service ontologies. For this purpose, the next section mainly describes the fundamental ideas of the proposed integrated process modeling methodology. A case study will also be given in this section to show the feasibility of our approach. Then, ontology-based process modeling technologies are studied. After that, the representation of the sample processes with the OWL-S service ontology is presented. Finally, some conclusions are summarized, followed by outlining of potential research topics. 


\section{PROCESS ANALYSIS: COMBINE ETOM AND ITIL}

\subsection{High-level Comparison between eTOM and ITIL}

While both the eTOM and ITIL are process frameworks, they are quite different in several aspects. As a standardized business process framework proposed by TMF, whose major contributors are Telecos enterprises, eTOM lays much more emphasis on the efficient and guaranteed provision of end-to-end service. This principle directly leads to the top-down hierarchical view of business processes, accompanied with detailed catalogue and descriptions of the compositional process elements, within the whole enterprise. When it comes to ITIL, things are somewhat different. ITIL is not that specific in classifying and decomposing its processes into more granular units, while on the other hand, it only provides guidance, or sometimes advices, on the implementations of the IT Service Management.

\subsection{Combine eTOM and ITIL Process Frameworks}

In spite of the conspicuous distinctions, different terminologies, and essentially complementary nature of ITIL and eTOM, there are still large overlaps between them. It has been widely recognized that companies involved in both frameworks will benefit most from the combination of the two approaches. Thus, recently there has been a heated discussion around the world about the integration of these two de-facto process frameworks. In fact, TMF also provides officially a fundamental integration approach, by mapping ITIL on to an eTOM enabled process environment [3]. Basically, the idea of this mapping is to illustrate how key ITIL scenarios (presently, only 3 scenarios in Change Management and Incident Management) are supported by eTOM elements. However, with no considerations on how to improve on the flow to ensure that it is fully compliant with the required ITIL process, this mapping is at best illustrative. In practice, the analysis of the diverse business processes within an enterprise is all-consuming; therefore, an iterative methodology [4] is more favorable and efficacious to gain a streamlined and integrated business process environment. As for the proposed iterative modeling methodology, a whole business process modeling lifecycle is divided into several rounds, with each round comprised of four phrases, as Figure 1 shows.

1. Firstly, model the concerned business process against the eTOM framework, which means the analysis, selection and composition of eTOM process elements in a topdown fashion (from eTOM level 1 down to level 3).

2. Then, in order to improve the soundness and robustness of the interim process previously gained, a comprehensive analysis is carried out with the help of the ITIL framework.

3. Next, it comes to a point that we verify the resulting business process against our original requirements.

4. If the verification shows that the modeled process is not that satisfactory, another round is initiated, starting from a more comprehensive requirement analysis. 


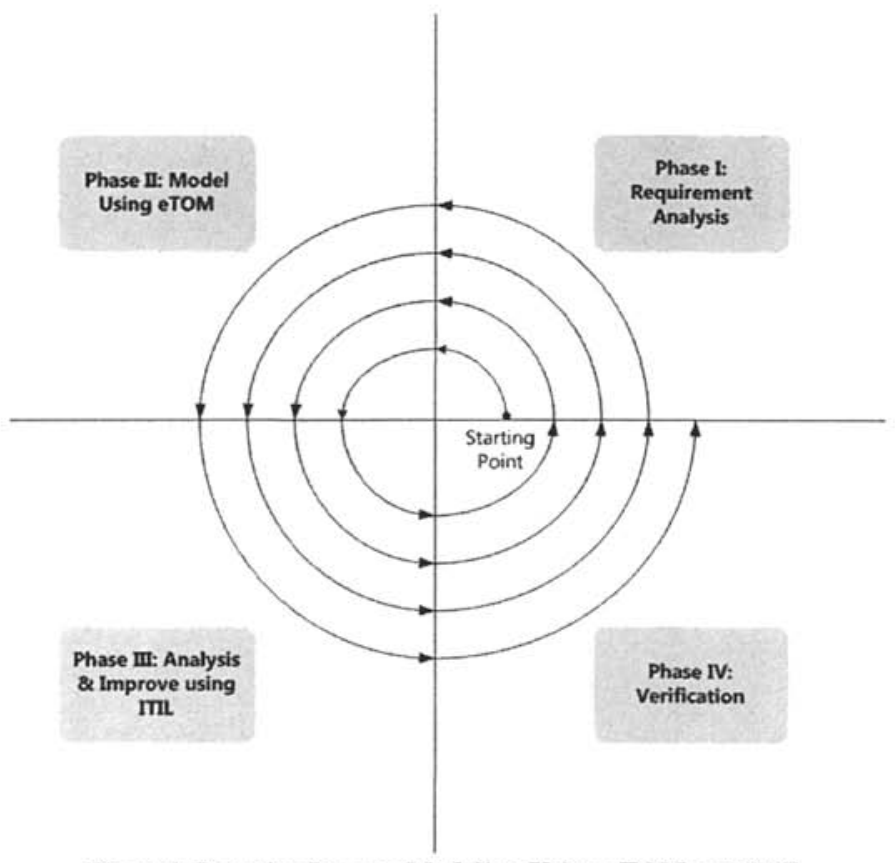

Figure1. Iterative Process Modeling Using eTOM and ITIL

\subsection{Case Study: Problem Resolution Process}

To illustrate the concepts described above, a detailed case study is provided in this subsection. In this case, a simplified problem resolution process is analyzed by using the elements in eTOM assurance process grouping, and later refined by ITIL. This process is initialized by the customer with some problematic services (such as the degradation of service QoS, or even a service failure) and finally resolved by the service provider. Figure 2 shows the modeled process and the analysis of it against ITIL (in this case, Incident Management in ITIL).

In fact, this correlation not only allows service provider to demonstrate compliance to both eTOM and ITIL frameworks, but also helps service providers to identify whether the process flow is deficient and where. This again allows service providers to refine and improve their business processes. For the problem resolution processes presented above, Figure 3 illustrates how ITIL sub processes can be used to enhance its quality. In this case, two ITIL sub processes are added to the interim process flow:

- Check for Known Errors: The service provider directly search its knowledge base for known errors, which could accelerate the whole problem resolution process. 


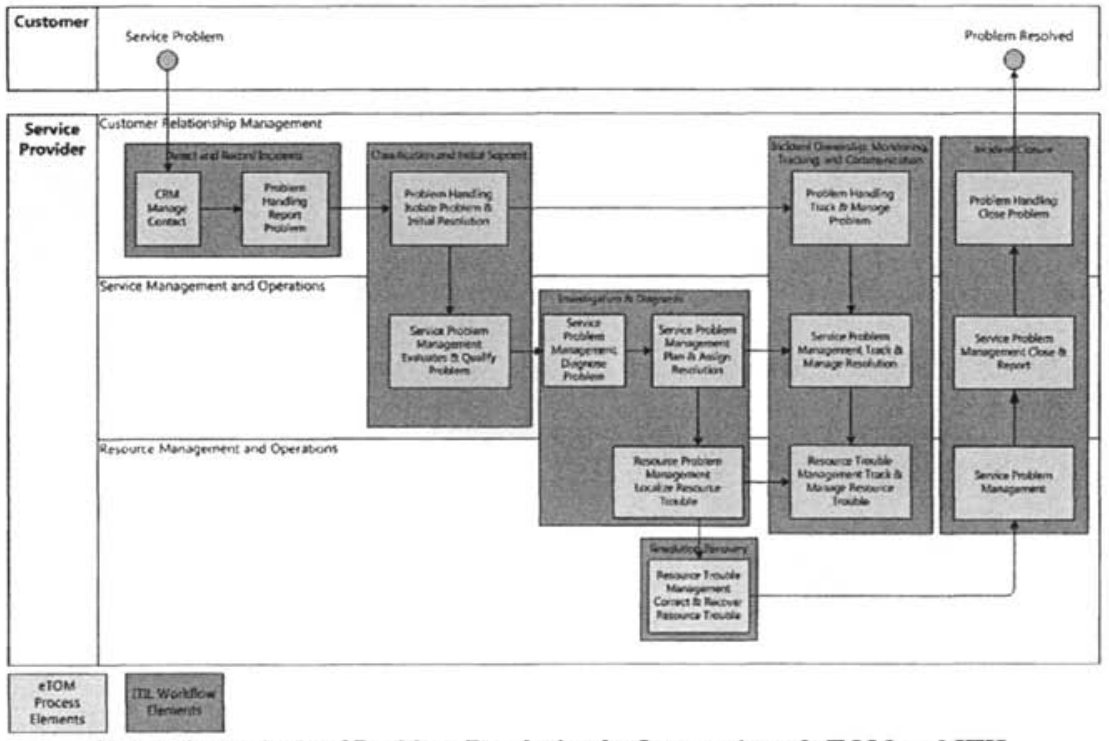

Figure 2. Analysis of Problem Resolution by Integration of eTOM and ITIL

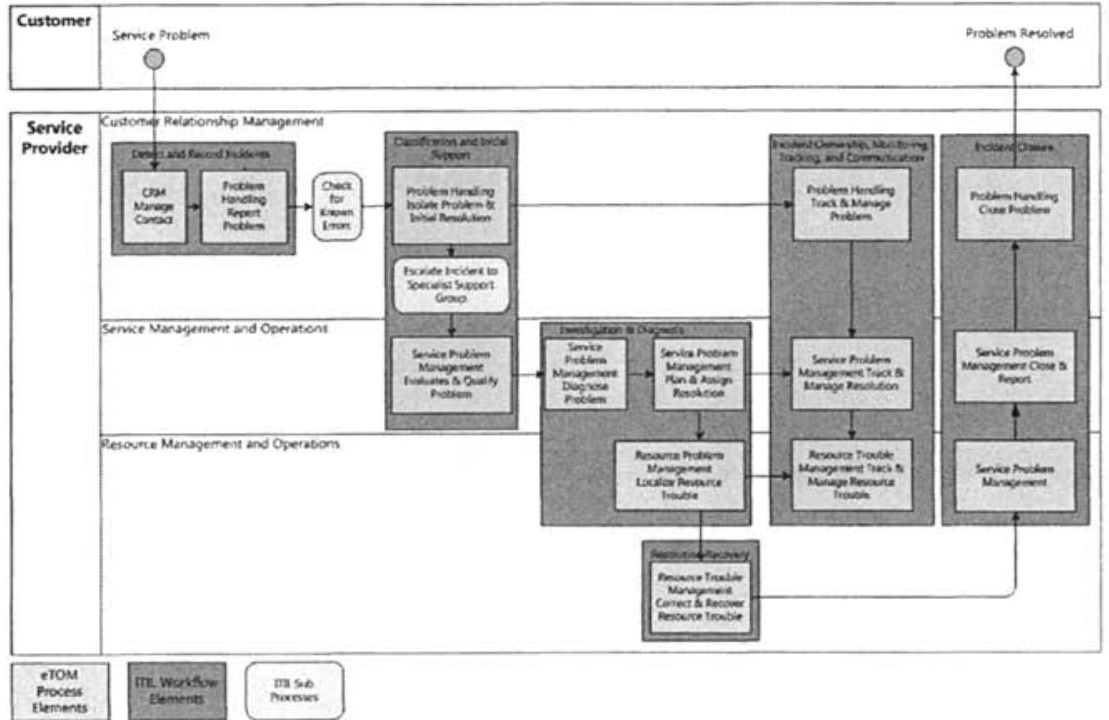

Figure 3. Modified Problem Resolution Process

- Escalate Incident to Specialist Support Group: Raise the incident to Specialist Support Group, which is composed of domain experts experienced and erudite enough to find quicker resolutions. 
In this way, it is convincing that the hybrid business process takes the best of both frameworks to provide a more streamlined end-to-end process flow.

\section{ONTOLOGY-BASED PROCESS MODELING}

Once the business processes are comprehensively analyzed by employing the preceding approach of integrating eTOM with ITIL, we are then faced with the problem of which process representation formalism to adopt to express the process details in a technical level. Presently, there are various formalisms, such as Business Process Execution Language for Web Services [5], XML Process Definition Language [6], Business Process Modeling Language [7], Business Process Specification Schema [8] and so forth, suited or intended for process/workflow representation being devised out of different perspectives. In [9], these formalisms are intensively analyzed and compared with each other according to common assessment metrics. There is also a prevailing trend towards a pattern-based analysis, such as [10] and [11], or ontology-based analysis, such as [12], of these formalisms. However, these researches are simply more concerned with the functional aspects shared by all process/workflow representation formalisms and sometimes it is unjustifiable to draw the conclusion that BPEL4WS is superior to XPDL, or vice versa.

In fact, these highly praised process representation techniques have not eliminated the huge semantic gap long existed between each other, hindering the interoperability between systems adopting different formalisms. Thus, a strategic approach which settles on some sort of "world language" and facilitates the common understanding between different parties involved in the business process is badly needed. In this section, a short introduction of emerging Semantic Web Service and Ontology technologies are given, followed by a brief description of the OWL-S process representation, which form the solid theoretical ground of the ontology-based business process modeling in this paper. Afterwards, we would use OWL-S for the description of the sample process presented in the preceding case studying section.

\subsection{Ontology and Semantic Web Service for Business Process Modeling}

The semantic web area [13] comprises a set of technologies to change current web from a network of contents and services interpreted and used by humans to a network in which such contents and services can be exploited by software agents. Among these technologies, the use of ontologies plays the most significant and active role. An ontology is defined as "a formal specification of a shared conceptualization [14]". Practically speaking, an ontology is a hierarchy of concepts, attributes, constraints, and relations that defines a common vocabulary in a specific domain, stressing uniform knowledge representation and knowledge sharing between heterogeneous systems.

Semantic Web Service is a particularly thriving area within semantic web technologies. Their objective is to provide a set of functionalities that can be understood and interpreted by software systems to exploit (discovery, composition, invocation) these functionalities in an automatic manner. To achieve this goal, a set of 
ontologies have been defined that allows the description of these functionalities. Among all these proposals, the most relevant are OWL Services (OWL-S), Web Service Modeling Ontology (WSMO), and Semantic Web Services Ontology (SWSO) [15]. Although the modeling facilities provided by each representation are not so similar, all of them share a similar semantics (in terms of IOPE ${ }^{1}$ ). [16] and [17] both pointed out, though from slightly different perspectives, three key semantics required for ontology-based process/workflow management, which can be summarized as follows:

- Process Element Ontology (PEO): A business process is composed of a set of ordered process elements represented in ontologies. Each PEO contains machine understandable information about the service.

- Control Flow between PEOs: This kind of semantic is responsible for the ordered (for example, sequence, split, if-else-then, and so on) execution of compositional PEOs.

- Data Flow between PEOs: In order to represent the semantic information passing between different PEOs during the period of process/workflow execution.

In the next subsection, we would give an deeper analysis of OWL-S based on these common semantic requirements.

\subsection{OWL-S Process Representation}

OWL-S [18] is a set of ontologies, written in OWL, which can be used to describe (at a higher/more detailed semantic level) what a service does and how it interacts with other services. The OWL-S ontology defines a Service class as the central class for describing what the service requires and provides for the users. To represent interactions, the ServiceModel class and its subclass Process have been defined. They are based on existing techniques for workflow and process modeling. In this context, two kinds of processes can be distinguished: atomic processes and composite processes.

An atomic process is a description of a service that expects one (possibly complex) message and returns one (possibly complex) message in response. Thus, this type of processes can be executed directly. Each AtomicProcess class has a Grounding information associated to it, allowing a client to build and interpret the messages interchanged with the service.

A composite process is one that maintains some state; each message the client sends advances it through the process. This composition can be achieved through the adoption of various control structures: sequence, split, split and join, any-order, choice, if-then-else, iterate, repeat-while, and repeat-until. Another specific characteristic of composite processes is the data flows. Whereas in an atomic process inputs are generated by a client and outputs are generated by the process, in a composite process, inputs can originate from a client or another process, and outputs can be generated by different processes.

In order to tie a bunch of processes together, we also need to know their preconditions for execution and any side-effects they might have. OWL-S has

${ }^{1}$ IOPE stands for "Input, Output, Prediction, and Effect". 
hasPrecondition and haseffect to represent preconditions and effects and these are in fact the "Control Flow between PEOs" mentioned in the preceding subsection. As for the data flow between POEs, OWL-S requires that service inputs and outputs be typed with a class of the related domain ontology.

With these representation facilities, it is possible to achieve the goal of creating a complex and machine-comprehensible description of composite business processes which can be shared by heterogeneous systems.

\subsection{Case Study: Problem Resolution Process in OWL-S}

To illustrate the concepts described above, we would represent the sample business process previously studied and analyzed using the integrated approaches of eTOM and ITIL proposed earlier in this paper. For this, each process element, such as "Problem Handling Report Problem" and "Check for Known Errors" is defined as a an atomic process which takes in input and returns output. In our sample process, most of the process elements are executed sequentially, so process:Sequence suffices to represent this execution order. When it comes to other situations, thing are much different.

- Split: This execution pattern contains a bag of process components to be executed concurrently. In our sample process, "Resource Problem Management Localize Resource Trouble" bifurcates (splits) into two branches, say "Resource Trouble Management Resource Trouble" and "Resource Trouble Management Correct \& Recover Resource Trouble", to execute concurrently. Therefore, process:Split can be employed to define this scenario.

- Split and Join: This execution pattern consists of concurrent execution of process components with barrier synchronization. In this case, "Problem Handling Isolate Problem \& Initial Resolution" first splits into two concurrent process, "Problem Handling Track \& Manage Problem" and "Escalate Incident to Specialist Support Group", and finally "joins" to be the precondition of process "Service Problem Management Track \& Manage Resolution". For this scenario, process:Split-Join can be used.

\section{CONCLUSIONS AND FUTURE WORK}

With accelerated shifting trends from device-oriented to process-oriented service management, large portions of business organizations are now on their way of managing their diverse business and operational processes. Moreover, there is an emerging requirement for the integration and interoperability between heterogeneous BPMS. Against this background, the methodology of ontology-based business process modeling using eTOM and ITIL developed in this paper combines eTOM and ITIL as the hybrid analysis framework and builds on ontology-driven information integration to address the need for a theoretically sound basis for modeling composite end-to-end business processes. 
As we observed previously in this paper, there are still much to do to reinforce the proposal in this paper. Presently, we only define each process element as atomic process, with no information (parameters in the $\mathrm{PEO}$ ) indicating what PEOs provide and return. To address this problem, other aspects in NGOSS and ITIL concerning actual data/message transfer between process elements must be further explored into detail. Another potential research topic might be the expression of preconditions and effects in OWL-S. Currently, OWL-S does not define a default language to represent such logic formulas. However, it recommends and provides some facilities to work with the Semantic Web Rule Language [19], and gives a mechanism to represent those formulas in other languages. Moreover, defining business process is only the first step towards the vision of BPMO (Business Process Management Ontology), a unique business process management ontology, much work still needs to be done to achieve this visionary goal.

\section{REFERENCES}

1. TeleManagement Forum, Enhanced Telecom Operations Map (eTOM) - The Business Process Framework (2007).

2. J. Gialelis, A. Kalogeras, C. Alexakos, M. Georgoudakis, and S. Koubias, Advanced Enterprise Process Modeling Utilizing Ontology Semantics, in Proc. of 1Oth IEEE International Conference on Emerging Technologies and Factory Automation ETFA (2005), pp.589-595.

3. TeleManagement Forum, Enhanced Telecom Operations Map (eTOM) - The Business Process Framework, Application Note V: An Interim View of an Interpreter's Guide for eTOM and ITIL Practitioners (2005).

4. C. Larman, Agile and Iterative Development: A Manager's Guide (Addison-Wesley Professional: 2003).

5. T. Andrews, F. Curbera, H. Dholakia, Y. Goland, J. Klein, F. Leymann, K. Liu, D. Roller, D. Smith, S. Thatte, I. Trickovic, and S. Weerawarana. Business Process Execution Language for Web Services VersionI.I, Technical report, OASIS (2003).

6. Workflow Management Coalition, Process Definition Interface - XML Process Definition Language, Workflow Management Coalition Workflow Standard WFMC-TC-1025, Version2.0 (2005).

7. Arkin, Business process modeling language. Draft, BPMI (2002).

8. UN/CEFACT and OASIS, Business Process Specification Schema. Technical report (2001).

9. V.A. Danciu and A. Vitalian, Formalisms for IT management process representation, in First IEEE/IFIP International Workshop on Business-Driven IT Management, BDIM 2006, v 2006, First IEEE/IFIP International Workshop on Business-Driven IT Management, BDIM (2006), pp.45-54.

10. P. Wohed, W. Aalst, M. Dumas, and A. Hofstede, Pattern Based Analysis of BPEL4WS, Technical Report FIT-TR-2002-04, QUT (2002).

11. S. White, Process Modeling Notations and Workflow Patterns, BPTrends (2004). http://www.bptrends.com (Accessed May 4, 2007). 
12. V. Gábor, B. Andersson, and P. Wohed, An Ontology Based Analysis of BPEL4WS and WSCI, in Proc. of the 3rd Nordic Conference on Web Services (NCWS 2004), ISBN 91 7636-431-3(2004).

13. T.B. Lee, J. Hendler, and O. Lassila, The Semantic Web, Scientific American. Volume 284, Number 5, pp.34-43, (2001).

14. T. Gruber, A Translation Approach to Portable Ontology Specifications, Knowledge Acquisition. Volume 5, Number 2, pp.199-220, (1993).

15. M. Burstein, C. Bussler, T. Finin, M. Huhns, M. Paolucci, A. Sheth, S. Williams, and M. Zaremba, A semantic Web services architecture, IEEE Internet Computing. Volume 9 , Number 5, pp.72-81, (2005).

16. H. Zhao and S. Shen, Web Services Workflow Management Based on OWL, Journal of Central South University (Science and Technology). Volume 36, Special 1, (2005).

17. J. Pathak, D. Caragea and V. Honavar, Ontology-extended component-based workflows: A framework for constructing complex workflows from semantically heterogeneous software components, in Leciure Notes in Computer Science, v 3372, Semantic Web and Databases - Second International Workshop, SWDB 2004 (2005), pp.41-56.

18. D. Martin, M. Burstein, J. Hobbs, O. Lassila, D. MeDermott, S. McIlraith, S. Narayanan, M. Paolucci, B. Parsia, T. Payne, E. Sirin, N. Srinivasan and K. Sycara, OWL-S: Semantic Markup for Web Services, W3C Member Submission (2004).

19. I. Horrocks, P. Patel-Schneider, H. Boley, S. Tabet, B. Grosof, and M. Dean, SWRL: A Semantic Web Rule Language Combining OWL and RuleML, W3C Member Submission (2004). 